\title{
Statistiques circulaires et utilisations en psychologie
}

\author{
Catherine Mello \\ Université de Montréal
}

\begin{abstract}
Researchers in psychology using angular or cyclic data are faced with problems of periodicity and arbitrariness, associated with circular statistics, that render usual methods of parameter calculation useless. This introduction to circular statistics presents trigonometric functions used in the computation of circular parameters such as center of mass, concentration, dispersion and homeward component. Common circular distributions and circular statistical inference methods that may be useful in psychology are also described. An example of a simple orientation experiment illustrates the application of these statistical tests using Microsoft Excel.
\end{abstract}

Le chercheur en psychologie traitant des données angulaires ou cycliques est confronté aux problèmes de périodicité et de l'arbitraire du système de mesure que posent les statistiques circulaires. Les méthodes usuelles de calcul de paramètres comme la moyenne ne sont alors d'aucune utilité. Cette introduction aux statistiques circulaires présente des fonctions trigonométriques permettant le calcul des paramètres circulaires: centre de masse, concentration, dispersion et homeward component. Des distributions circulaires couramment utilisées ainsi que des méthodes d'inférence statistique mises au point pour des mesures circulaires pertinentes à la psychologie sont également décrites. Un exemple d'une expérience simple d'orientation illustre l'application des différents tests statistiques au moyen du logiciel Microsoft Excel.

On retrouve dans différents domaines scientifiques des données mesurées en angles, se rapportant à l'orientation ou à la direction de certains phénomènes. Par exemple, les océanographes peuvent s'intéresser à la direction des courants maritimes; les météorologues à celle des vents et les géologues à l'orientation des cristaux de roches ignées (Fisher, 1993). Des biologistes peuvent considérer l'axe sur lequel l'abeille déploie sa danse en fonction de stimuli lumineux, ou la déviation du parcours de pigeons voyageurs par rapport à leur destination (Batschelet, 1981). Cependant, les mesures de direction ne sont pas les seules données circulaires: des périodes de phénomènes cycliques peuvent être représentées sous la forme de cercles de 360 degrés. Par exemple, on pourrait déterminer que pour un rythme biologique d'une période de 24 heures et de phase Oh (ou 24h), un événement $X$ se produisant à 3 h correspond à $45^{\circ}$. De même, une représentation circulaire peut être utilisée pour exprimer la fréquence de séismes en fonction du mois, sur une période d'un an.

La recherche en psychologie utilise également des données circulaires, le plus souvent calculées à partir du système géographique. Le comportement humain dans des expériences d'orientation (telles que celle de Montello, Lovelace, Golledge et Self, 1999, où les participants doivent indiquer la direction du point de départ à différents moments du parcours) peut être traité comme celui des pigeons voyageurs mentionné plus haut. L'étude que font les neuropsychologues des rythmes circadiens, tels que les fluctuations de mélatonine, se prête également à des analyses conçues pour des données circulaires.

Les mesures d'angle (ou les mesures cycliques représentées par des angles) sont considérées comme des données circulaires car elles se distribuent entre 0 et 360 degrés. Il s'ensuit que $0^{\circ}$ et $360^{\circ}$ sont équivalents, tout comme 0 h et $24 \mathrm{~h}$. Les données linéaires usuelles, telles que des mesures de distance ou de masse, occupent toute la droite réelle. Par ailleurs, le point d'origine (la direction $0^{\circ}$ ) et le sens de rotation des mesures circulaires sont arbitraires (Jammalamadaka \& SenGupta, 2001). En mathématiques, les angles sont mesurés dans le sens anti-horaire à partir du 
point $(1,0)^{1}$ d'un cercle. Dans certaines situations mentionnées précédemment, il est probable que les angles aient été mesurés à partir du nord (géographique ou magnétique, de coordonnées cartésiennes $(0,1))$, dans le sens horaire (Batschelet, 1981). De tels angles sont nommés azimuts. La Figure 1 illustre bien comment l'adoption de ces deux systèmes répandus de mesure angulaire, que nous nommerons mathématique et géographique, attribuent une valeur différente à une même direction.

Figure 1 : Une même direction mesurée selon les systèmes mathématique et géographique.

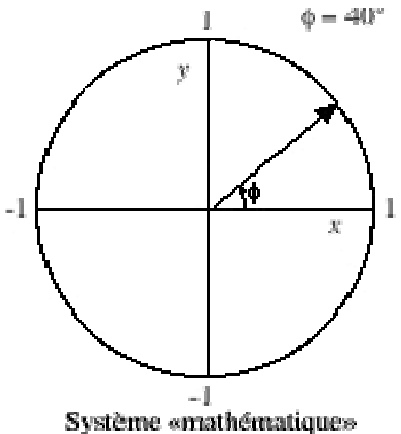

Systène wmathematiques

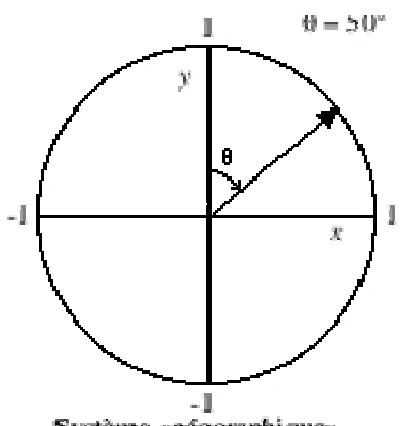

Systêne :gGographique*
L'obstacle principal posé par la circularité des données à l'application de techniques statistiques usuelles, conçues pour des données linéaires, réside dans ce choix arbitraire de la direction zéro et du sens de rotation. Le système de mesure angulaire choisi influence directement la valeur de différents moments statistiques (p. ex., moyenne, écart type). Les problèmes rencontrés dans le calcul de la moyenne arithmétique seront illustrés plus loin. Les méthodes statistiques basées sur le rang ne peuvent s'appliquer: le fait qu'un angle soit «supérieur» à un autre dépend du choix de l'origine et du sens de rotation. La périodicité des mesures circulaires - soit le fait que l'origine $\left(0^{\circ}\right)$ coïncide avec la fin $\left(360^{\circ}\right)$ complique également le calcul de la distance entre deux points. Il devient alors nécessaire d'adopter des techniques d'analyses invariantes au choix de l'origine et du sens de rotation et permettant de parer au problème de périodicité.

Cependant, toutes les données mesurées en angles ne sont pas circulaires: lorsqu'elles sont circonscrites à une région précise du cercle, le problème de la périodicité ne se présentant pas, elles peuvent être traitées comme des données linéaires. Soit une thèse d'Honneur où les participants doivent indiquer la position de villes européennes par rapport à des villes nord-américaines: à moins qu'ils ignorent où se situent ces continents, les

1 Coordonnées cartésiennes, aussi appelées coordonnées rectangulaires. azimuts devraient se situer entre $0^{\circ}$ et $180^{\circ}$. Par contre, s'il s'agissait de villes européennes par rapport à des villes africaines, la chercheuse serait confrontée à la difficulté de comparer des réponses comme $350^{\circ}$ et $10^{\circ}$.

Si des angles individuels peuvent être ou non des données circulaires, il en est de même pour l'écart entre deux angles. Quand un individu doit indiquer la direction de Montpellier (France) par rapport à Alger (Algérie), la direction réelle $\theta_{0}$ serait de $0^{\circ}$ ou $360^{\circ}$. Pour des raisons théoriques, la chercheuse pourrait s'intéresser au sens des erreurs (erreur $=\theta_{0}-\theta$ ): une erreur de $-20^{\circ}$ signifierait que l'individu croit que Montpellier est plus à l'ouest; tandis qu'une erreur positive signifierait un biais vers l'est. L'erreur est alors une mesure circulaire, dépendante du choix du sens de rotation, tandis que l'imprécision (l'erreur sans égard au sens) n'en est pas une.

\section{Notions élémentaires de géométrie plane}

Soit un point $\mathrm{P}$, situé sur un plan, dont la position peut être déterminée par les coordonnées cartésiennes $x$ et $y$. Il peut également être défini par un angle $\phi$. Soit aussi un vecteur coïncidant avec l'axe $X$ positif ayant une direction zéro, appelé l'axe polaire. Si nous faisons subir au vecteur une rotation antihoraire jusqu'à ce qu'il passe par $\mathrm{P}$, l'étendue de cette rotation est l'angle $\phi$. Bien que des logiciels comme SPSS et Microsoft Excel utilisent les radians, les degrés seront ici préférés car les angles ainsi exprimés sont plus facilement visualisés par le lecteur. Pour convertir des radians en degrés ou inversement, il suffit de se rappeler l'équation suivante:

$$
360^{\circ}=2 \pi \mathrm{rad}
$$

La distance entre $\mathrm{P}$ et l'origine, notée $r$, et l'angle $\phi$ constituent les coordonnées polaires de P.

Figure 2 : Coordonnées cartésiennes et polaires de P.

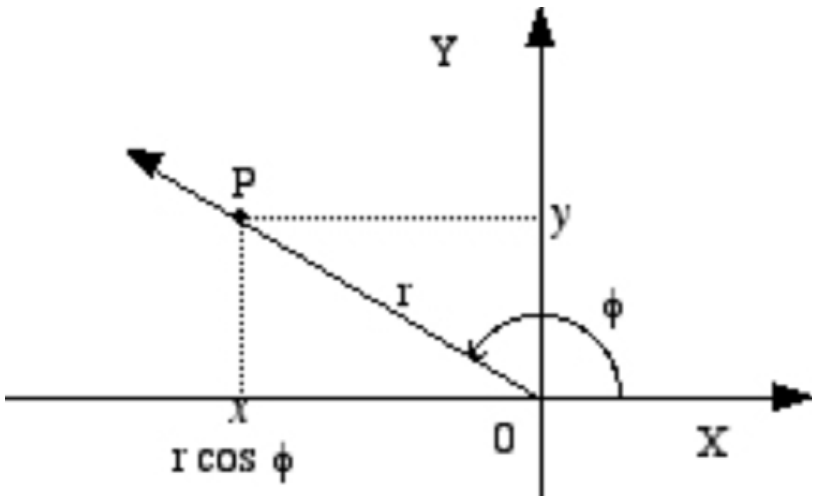

Pour convertir des coordonnées polaires en coordonnées cartésiennes:

$$
\begin{aligned}
& x=\mathrm{r} \cos \phi \\
& y=\mathrm{r} \sin \phi
\end{aligned}
$$

Pour convertir des coordonnées cartésiennes en 
coordonnées polaires:

Pour évaluer r:

$$
\begin{gathered}
x^{2}+y^{2}=r^{2}\left(\cos ^{2} \phi+\sin ^{2} \phi\right)=r^{2} \text { et } \\
r=\left(x^{2}+y^{2}\right)^{1 / 2}
\end{gathered}
$$

Pour évaluer $\phi$ :

$$
\tan \phi=\frac{y}{x}=\frac{r \sin \phi}{r \cos \phi}=\frac{\sin \phi}{\cos \phi}
$$

Donc, $\phi=\arctan (y / x)$. Cependant, puisqu'il est convenu que la fonction arc tan a comme image $]-90^{\circ}, 90^{\circ}[$, il faut être attentif aux signes de $x$ et $y$ pour bien déterminer l'angle.

Ainsi,

$$
\begin{gathered}
\phi=\arctan (y / x) \text { lorsque } x>0 \text { et } y \geq 0 \\
\phi=\arctan (y / x)+180^{\circ} \text { lorsque } x<0 \\
\phi=90^{\circ} \text { Lorsque } x=0 \text { et } y>0 \\
\phi=270^{\circ} \text { Lorsque } x=0 \text { et } y<0 \\
\phi=\arctan (y / x)+360^{\circ} \text { Lorsque } x>0 \text { et } y<0 \\
\phi=\text { indéterminé lorsque } x=0 \text { et } y=0
\end{gathered}
$$

Ces différentes équations, qui paraissent dénuées de sens pour le lecteur étudiant en psychologie, se révéleront utiles dans le calcul de paramètres tels que la moyenne. Dans les exemples qui suivront, la direction étant la seule variable d'intérêt, seule la valeur de $\phi$ sera donnée, tandis qu'une valeur de 1 sera attribuée par défaut à $r$. Batschelet (1981) fournit davantage de détails sur les fonctions trigonométriques ainsi que des analogies entre les statistiques circulaires et linéaires.

\section{Paramètres circulaires}

\section{Direction moyenne}

Lorsque le chercheur cherche à définir la direction moyenne, ou direction préférée par les participants dans une tâche, il ne peut simplement calculer la moyenne arithmétique des angles. Étant donné la nature circulaire des données, il pourrait se retrouver avec des résultats aberrants, comme en témoignent les deux exemples suivants (inspirés de Jammalamadaka \& SenGupta, 2001).

Figure 3: Périodicité et calcul de la moyenne arithmétique.

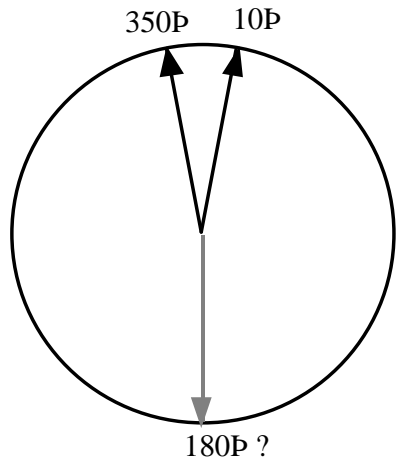

Exemple 1: Problème de la périodicité des données.

Soit deux participants ayant fourni des azimuts de $10^{\circ}$ et $350^{\circ}$ en réponse à la question suivante: «Quelle est la direction de Montpellier (France) par rapport à Alger (Algérie)?». Il est clair que la direction moyenne devrait se trouver à $0^{\circ}$, soit le nord géographique. Pourtant, la moyenne arithmétique calculée à partir de ces données serait de $180^{\circ}$, soit le sud!

Exemple 2: Problème du choix arbitraire de l'origine et du sens de rotation.

Soit quatre participants laissés au centre d'un boisé, ayant comme consigne de retrouver le laboratoire de psychologie. Leurs directions de départ, calculées avec le système mathématique sont: $50^{\circ}, 160^{\circ}, 210^{\circ}$ et $300^{\circ}$. La moyenne arithmétique indique une direction moyenne de $180^{\circ}$, ce qui représente l'ouest. Par contre, si ces mêmes directions étaient calculées avec le système géographique, nous obtiendrions des azimuts respectifs de $40^{\circ}, 290^{\circ}, 260^{\circ}$ et $140^{\circ}$. La moyenne arithmétique serait encore de $180^{\circ}$, mais elle représenterait cette fois le sud...

La transcription de $350^{\circ}$ en un azimut équivalent de $-10^{\circ}$ aurait résolu le problème de l'exemple 1, mais il n'existe pas de solution aussi simple lorsqu'un grand nombre de directions variées est pris en compte. La méthode appropriée pour aborder de la direction moyenne est le calcul du centre de masse, qui peut être obtenu par l'algèbre vectorielle ou les fonctions trigonométriques. La première méthode consiste à additionner les vecteurs de directions individuelles $\vec{e}_{i}$, produisant un vecteur de longueur $R=$ $\left|\sum \vec{e}_{i}\right|$, ensuite divisé par le nombre de vecteurs (voir la Figure 4 pour une schématisation de la démarche) pour fournir un vecteur moyen $\vec{m}$. La longueur $r$ de ce dernier sera expliquée à la section suivante.

Figure 4: Calcul du vecteur moyen $m$ de l'exemple 2 au moyen d'un polygone vectoriel.
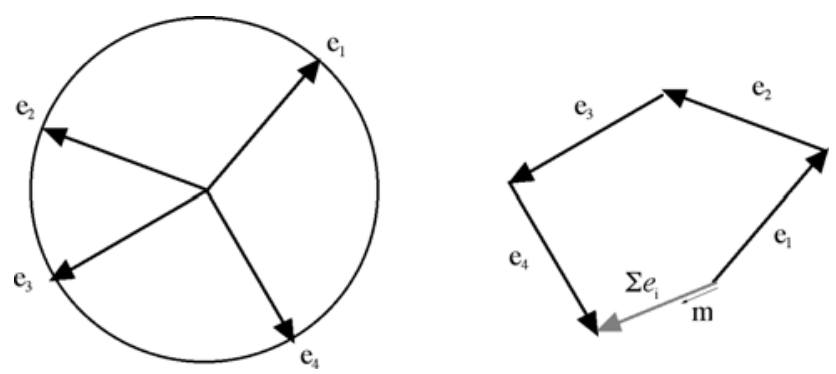

Il est par contre plus aisé, au moyen de différents logiciels statistiques, d'utiliser la démarche équivalente avec les fonctions trigonométriques. Chacun des vecteurs $\overrightarrow{\boldsymbol{e}}_{i}$ possède les coordonnées $x_{i}$ et $y_{i}$, ainsi calculées:

$$
x_{\mathrm{i}}=\cos \phi_{\mathrm{i}} \text { et } y_{\mathrm{i}}=\sin \phi_{\mathrm{i}}
$$

Pour calculer les coordonnées cartésiennes du centre de 
masse:

$$
\bar{x}=\frac{1}{n}\left(x_{1}+x_{2}+\ldots+x_{n}\right) \text { et } \bar{y}=\frac{1}{n}\left(y_{1}+y_{2}+\ldots+y_{n}\right)
$$

De sorte que:

$$
\begin{gathered}
\bar{x}=\frac{1}{n}\left(\cos \phi_{1}+\cos \phi_{2}+\ldots+\cos \phi_{n}\right) \text { et } \\
\bar{y}=\frac{1}{n}\left(\sin \phi_{1}+\sin \phi_{2}+\ldots+\sin \phi_{n}\right)
\end{gathered}
$$

La longueur $r$ du vecteur moyen est ainsi obtenue:

$$
r=\left(\bar{x}^{2}+\bar{y}^{2}\right)^{1 / 2}
$$

Le calcul de l'angle moyen doit être adapté en fonction du quadrant où se trouve le vecteur moyen, en utilisant les Équations 5 à 9. Pour un vecteur situé dans le premier quadrant:

$$
\bar{\phi}=\arctan (\bar{y} / \bar{x})
$$

L'angle moyen ainsi calculé reflète alors le centre de masse des données sans être affecté par le choix de l'origine ou du sens de rotation propre au système de mesure angulaire.

\section{Mesures de concentration et de dispersion}

Tout aussi importante que la valeur de l'angle moyen est la concentration des directions individuelles autour du vecteur moyen. Avec des données unimodales, celle-ci est fournie par $r$, qui décroît quand les points se dispersent davantage autour du vecteur moyen, $r=1$ signifiant que toutes les données indiquent la même direction. Cependant, des échantillons plus petits favorisent des valeurs de $r$ plus grandes (voir Batschelet, 1981, pour une démonstration). À partir de $r$, il est possible d'obtenir la variance angulaire et l'écart type angulaire:

$$
s^{2}=2(1-r) \text { et } s=[2(1-r)]^{1 / 2}
$$

L'écart type angulaire étant donné en radians, il doit être multiplié par $180^{\circ} \%$ pour le convertir en degrés.

Le homeward component, utilisé dans des expériences où des animaux doivent retrouver un point donné (leur nid, par exemple), peut être une mesure de dispersion pertinente à certaines tâches d'orientation propre à la psychologie. En plus de connaître la concentration des données autour de la direction moyenne, le chercheur s'intéresse alors à l'écart entre celle-ci et la direction attendue. Cette information est fournie par $v$, lorsque le vecteur moyen $m$ a un angle $\phi$ et une concentration $r$, pour une direction attendue $\theta_{0}$ :

$$
v=r \cos (\bar{\phi}-\theta 0)
$$

Ainsi, $v$ prend une valeur de 1 lorsque tous les individus se dirigent ou pointent dans la direction attendue, et devient négatif lorsque l'écart entre les directions moyenne et attendue dépasse $90^{\circ}$. Des tests d'hypothèses basés sur $v$ permettent de vérifier si la direction moyenne diffère de celle qui était prévue (test $V$ ). Toutefois, $v$ dépendant de nombre d'observations (tout comme $r$ ), les $v$ de deux échantillons de tailles différentes ne peuvent être comparés.
Un test non paramétrique permet par contre la comparaison d'échantillons quant à la déviation par rapport à la direction prévue (test $U$ de Mann-Whitney sur la déviation angulaire).

\section{Distributions circulaires}

\section{La distribution uniforme}

Cette distribution sert de modèle pour le hasard: lorsque toutes les directions ont autant de chance d'être choisies (Fisher, 1993) et donc qu'aucun secteur du cercle n'est préféré, la distribution circulaire est dite uniforme. Elle est l'équivalent de la distribution uniforme en statistiques linéaires où:

Quand $x$ prend des valeurs entre $a$ et $b$ :

$$
f(x)=\frac{1}{b-a}
$$

Donc, pour $\phi$ entre 0 et $2 \pi$ :

$$
f(\phi)=\frac{1}{2 \pi}
$$

Le centre de cette distribution est donc l'origine 0 et aucune direction moyenne n'est définie.

\section{La distribution von Mises}

La distribution von Mises est la plus fréquemment utilisée dans l'analyse de directions (Bastchelet, 1981) et joue, en statistiques circulaires, un rôle similaire à la distribution normale usuelle. On la nomme d'ailleurs aussi distribution circulaire normale, car elle s'apparente à la loi normale linéaire en regard des éléments suivants:

- elle dépend de deux paramètres: la direction moyenne $\mu$ et la concentration autour de la moyenne $\kappa$;

- elle est symétrique par rapport à la direction moyenne $\mu$

- elle est unimodale et son mode correspond à la direction moyenne $\mu$.

La fonction de densité2: de la loi von Mises, où $0<\mu<2 \pi$ et $\kappa \geq 0$, pour $0<\phi<2 \pi$ :

$$
f(\phi ; \mu, \kappa)=\frac{1}{2 \pi 0(\kappa)} e^{\kappa \cos (\phi-\mu)}
$$

\footnotetext{
${ }^{2} \mathrm{I}_{0}(\kappa)$ provient de la fonction de Bessel d'ordre 0:

$\mathrm{I} 0(\kappa)=\frac{1}{2 \pi} \int_{0}^{2 \pi} e^{\kappa \cos \phi} d \phi$
} 
Figure 5: Représentation graphique de distributions von Mises où $\mu=180^{\circ}$ et $\kappa=1$ ou 2 .

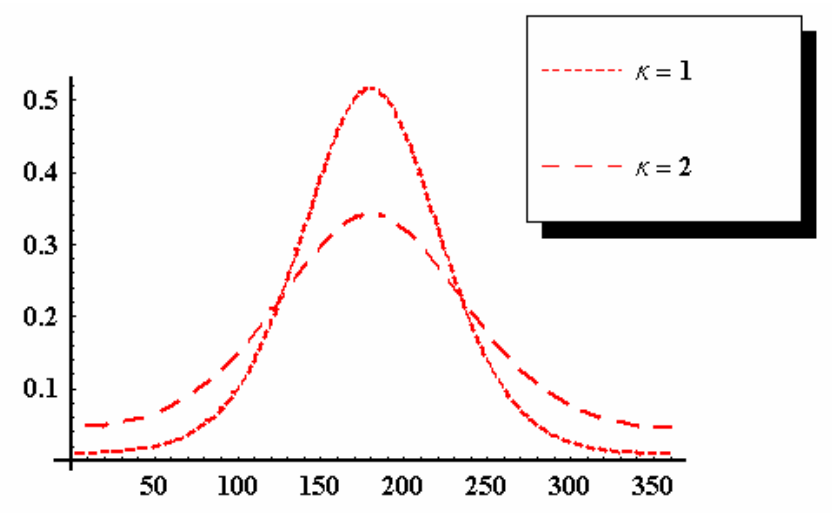

Inférence statistique sur des données circulaires

\section{Test de conformité de Kuiper}

Ce test est une adaptation du test de conformité de Kolmogorov-Smirnov, rendu invariant quant à l'origine et au sens de rotation du système de mesure angulaire. Il permet de vérifier si les données sont conformes à une distribution donnée, par exemple la distribution uniforme ou une distribution von Mises où $\mu$ et $\kappa$ sont connus.

$\mathrm{H}_{\mathrm{o}}$ : Les données proviennent d'une distribution théorique donnée.

$\mathrm{H}_{1}$ : Les données ne proviennent pas de la distribution théorique mentionnée en $\mathrm{H}_{0}$.

Comme c'est le cas dans le test Kolmogorov-Smirnov, il faut calculer écarts maximaux supérieurs $\left(D^{+}\right)$et inférieurs (D) entre les fréquences cumulées théoriques et observées (Fisher, 1993). On obtient ainsi la statistique $K$, qui entraîne un rejet de l'hypothèse nulle si elle dépasse $K(\propto)^{3}: K=n^{1 / 2} V_{n}$, où

$$
V_{n}=\mathrm{D}^{+}+\mathrm{D}^{-}
$$

Pour vérifier l'hypothèse nulle d'une distribution uniforme, il s'agit donc de placer les observations en ordre croissant, de telle sorte que $\phi_{1} \leq \phi_{2} \leq \ldots \leq \phi_{n}$. La fréquence cumulée théorique de chaque observation $\phi_{i}$ est calculée: dans le cas d'une distribution uniforme, celle-ci est donnée par $\phi_{i} / 2 \pi$. On calcule ensuite la fréquence cumulée observée de chaque $\phi$ i: celle de la $i^{i e ̀ m e}$ observation est de $i / n$ puisque les observations sont placées en ordre croissant. Les écarts maximaux sont ensuite calculés afin d'évaluer $V$ :

$$
\begin{aligned}
& \mathrm{D}^{+}=\max \left\{\frac{1}{n}-\frac{\phi 1}{2 \pi}, \frac{2}{n}-\frac{\phi 2}{2 \pi}, \ldots, 1-\frac{\phi_{n}}{2 \pi}\right\} \\
& \mathrm{D}^{-}=\max \left\{\frac{\phi}{2 \pi}, \frac{\phi 2}{2 \pi}-\frac{1}{n}, \ldots, 1 \frac{\phi n}{2 \pi}-\frac{n-1}{n}\right\}
\end{aligned}
$$

3 Une table des valeurs critiques $K(\propto)$ est disponible à la page 341 du livre de Batschelet (1981).
La valeur de $V$ ainsi obtenue permet le calcul de $K$, qui sera comparée à la valeur critique $K(\propto)$.

\section{Le test V}

Ce test permet de déterminer si les données observées tendent à s'agglomérer autour d'une direction prédéterminée $\theta_{0}$, et donc si la distribution diffère significativement de l'uniformité (ou du hasard). Bien qu'appliqué aux déplacement d'animaux (Batschelet,1981), il sert aussi pour des tâches d'orientation en psychologie.

$\mathrm{H}_{0}$ : La population est uniformément distribuée.

$\mathrm{H}_{1}$ : Les directions sont agglomérées autour de la direction hypothétique.

Ce test est basé sur le homeward component (v), qui est la projection du vecteur moyen $m$, de longueur $r$ et d'angle polaire $\bar{\phi}$ (Équation 16), sur la direction hypothétique $\theta_{0}$. La statistique $u$ est calculée à partir de $v$, puis comparée à la valeur critique $u(\propto)^{4}$ pour rejeter $\mathrm{H}_{0}$, si $u>u(\propto)$ :

$$
u=(2 n)^{1 / 2} v
$$

\section{Le test non paramétrique U de Mann-Whitney (adaptation par Wallraff, 1979)}

Ce test permet de décider si deux groupes diffèrent significativement quant à leur performance d'orientation, lorsqu'une direction réelle $\theta_{\circ}$ est connue (Batschelet, 1981).

$H_{\text {o: }}$ Les deux populations d'où sont issues les échantillons ne diffèrent pas quant à leur performance d'orientation.

$\mathrm{H}_{1}$ : Les deux populations diffèrent significativement quant à leur performance d'orientation.

Il s'agit de calculer la déviation angulaire entre chaque angle observé des deux échantillons $\left(\phi_{\mathrm{i}}, \psi_{\mathrm{i}}\right)$ et la direction réelle $\theta_{0}$ :

$\left|\phi_{i}, \theta_{0}\right|=\arccos \left[\cos \left(\phi_{i}-\theta_{0}\right)\right]$ et $\left|\psi_{i}, \theta_{0}\right|=\arccos \left[\cos \left(\psi_{i}-\theta_{0}\right)\right](25)$

Les distances angulaires des deux échantillons sont ensuite combinées et placées en ordre croissant. Lorsque les échantillons contiennent deux données égales, un rang est attribué aléatoirement à chacune d'entre elles. On calcule ensuite la somme des rangs $S_{1}$ et $S_{2}$ des échantillons. On effectue ensuite le test $U$ de Mann-Whitney à partir des statistiques $U_{1}$ et $U_{2}$ suivantes:

$$
U_{1}=S 1-\frac{n(n+1)}{2} \quad U_{2}=S_{2}-\frac{n(n+1)}{2}
$$

On compare la plus petite valeur de $U$ ( qui sera $U_{1}$ ou $\left.U_{2}\right)$ à $U(\propto)^{5}$. Lorsque $U$ est inférieure ou égale à la valeur critique, l'hypothèse nulle est rejetée et on conclut que les

${ }^{4}$ Une table des valeurs critiques $u(\propto)$ est disponible à la page 336 du livre de Batschelet (1981).

${ }^{5}$ Les valeurs critiques de $U$ du test statistique WilcoxonMann-Whitney peuvent être trouvées dans Lindgren (1968). 
deux échantillons diffèrent quant à leur déviation angulaire par rapport à la direction attendue (ou performance d'orientation).

\section{Exemples d'inférence statistique dans une tâche}

\section{d'orientation}

Dans le cadre d'une expérience portant sur diverses habiletés visuo-spatiales, 10 étudiants en sciences économiques sont convoqués au pavillon du département de psychologie. Ce bâtiment leur étant inconnu, l'expérimentatrice les accueille à l'entrée pour les accompagner jusqu'au laboratoire de recherche, mais leur fait effectuer quelques détours inutiles. Une fois rendus au laboratoire, leur première tâche est d'indiquer la direction de la porte d'entrée du pavillon au moyen d'une «boussole» en carton. Les réponses, obtenues au moyen du système «mathématique», sont transposées en radians dans un chiffrier Excel. On calcule ensuite les coordonnées cartésiennes de chacune des réponses en radians à partir des Équations 2. La coordonnée $x$ du vecteur moyen étant inférieure à 0, l'Équation 6 est utilisée pour calculer l'angle moyen, ensuite transposé en degrés. On peut également calculer le paramètre de concentration et l'écart type angulaire (en degrés).

Figures 6: Données brutes et calculs pour une tâche d'orientation.

\begin{tabular}{|c|c|c|c|c|}
\hline Participant & éponse & Réponse (rad) & $x$ & $\mathrm{y}$ \\
\hline 1 & 166 & 2.90 & -0.97 & 0.24 \\
\hline 2 & 113 & 1.97 & -0.39 & 0.92 \\
\hline 3 & 155 & 2.71 & -0.91 & 0.42 \\
\hline 4 & 217 & 3.79 & -0.80 & -0.60 \\
\hline 5 & 142 & 2.48 & -0.79 & 0.62 \\
\hline 6 & 160 & 2.79 & -0.94 & 0.34 \\
\hline 7 & 192 & 3.35 & -0.98 & -0.21 \\
\hline 8 & 224 & 3.91 & -0.72 & -0.69 \\
\hline 9 & 127 & 2.22 & -0.60 & 0.80 \\
\hline 10 & 171 & 2.98 & -0.99 & 0.16 \\
\hline$n=$ & 10 & & & \\
\hline Réponse $(\mathrm{rad})=$ & - & \multicolumn{3}{|c|}{ =RADIANS(Réponse) } \\
\hline$x=$ & - & \multicolumn{3}{|c|}{$=\cos (x i)$} \\
\hline$y=$ & - & \multicolumn{3}{|c|}{$=S I N(x i)$} \\
\hline $\mathrm{x}$ moyen $=$ & -0.81 & \multicolumn{3}{|c|}{$=(1 / n) * \operatorname{SUM}(x 1: x 10)$} \\
\hline y moyen $=$ & 0.20 & \multicolumn{3}{|c|}{$=(1 / n) * \operatorname{SUM}(y 1: x 10)$} \\
\hline Angle moyen $(\mathrm{rad})=$ & 2.90 & \multicolumn{3}{|c|}{$=$ ATAN $(y$ moyen $/ x$ moyen $)+$ RADIANS(180) } \\
\hline Angle moyen (degrés) = & 166.14 & \multicolumn{3}{|c|}{$=$ DEGREES(Angle moyen (rad)) } \\
\hline$r=$ & 0.83 & \multicolumn{3}{|c|}{$=\left(\left(x \text { moyen } \_\right)+(y \text { moyen^ } 2)\right)^{\wedge}(1 / 2)$} \\
\hline $\mathrm{s}(\mathrm{rad})=$ & 0.58 & \multicolumn{3}{|c|}{$=(2 *(1-r)) \wedge(1 / 2)$} \\
\hline s (degrés) = & 33.18 & \multicolumn{3}{|l|}{$=\mathrm{DEGREES}(\mathrm{s}(\mathrm{rad}))$} \\
\hline
\end{tabular}

\section{Test de conformité à la distribution uniforme}

Si on souhaite uniquement vérifier si les participants répondent ou non au hasard, sans égard à l'exactitude des directions données, on peut effectuer un test de Kuiper. En utilisant l'option «Sort» du menu «Data» d'Excel, les réponses sont placées en ordre croissant et se font attribuer un rang de 1 à 10 (i). On calcule alors les fréquences cumulées observée et théorique de chaque observation, qui permettent d'obtenir $\mathrm{D}^{+}$et $\mathrm{D}^{-}$. Le $V$ qui en résulte fournit un $K$ supérieur à la valeur critique de 1.37 (pour $n=10, \propto=.05$ ). On peut donc rejeter l'hypothèse nulle d'une distribution uniforme.

Figure 7: Calcul des statistiques nécessaires à un test de Kuiper sur l'uniformité.

\begin{tabular}{|c|c|c|c|c|c|c|}
\hline Participant & rad) & Rang & FC obs. & FC thé. & Écart sup. & Écart inf. \\
\hline 2 & 1.97 & 1 & 0.1 & 0.31 & -0.21 & 0.31 \\
\hline 9 & 2.22 & 2 & 0.2 & 0.35 & -0.15 & 0.25 \\
\hline 5 & 2.48 & 3 & 0.3 & 0.39 & -0.09 & 0.19 \\
\hline 3 & 2.71 & 4 & 0.4 & 0.43 & -0.03 & 0.13 \\
\hline 6 & 2.79 & 5 & 0.5 & 0.44 & 0.06 & 0.04 \\
\hline 1 & 2.90 & 6 & 0.6 & 0.46 & 0.14 & -0.04 \\
\hline 10 & 2.98 & 7 & 0.7 & 0.48 & 0.23 & -0.13 \\
\hline 7 & 3.35 & 8 & 0.8 & 0.53 & 0.27 & -0.17 \\
\hline 4 & 3.79 & 9 & 0.9 & 0.60 & 0.30 & -0.20 \\
\hline 8 & 3.91 & 10 & 1 & 0.62 & 0.38 & -0.28 \\
\hline$n=$ & 10 & & & & & \\
\hline FC observée $=$ & - & \multicolumn{5}{|c|}{$=\mathrm{i} / \mathrm{n}$} \\
\hline FC théorique $=$ & - & \multicolumn{5}{|c|}{$=$ réponse $(\mathrm{rad}) /(2 * \mathrm{PI}())$} \\
\hline Écart supérieur= & - & \multicolumn{5}{|c|}{$=F C$ observée- $F C$ théorique } \\
\hline Écart inférieur $=$ & - & \multicolumn{5}{|c|}{$=F C$ théorique- $(F C$ observée- $(1 / n))$} \\
\hline$D+=$ & 0.38 & \multicolumn{5}{|c|}{$=\operatorname{MAX}($ Écart sup. $1:$ Écart sup.10) } \\
\hline$D-=$ & 0.31 & \multicolumn{5}{|c|}{$=\operatorname{MAX}($ Écart inf. $1:$ Écart inf. 10) } \\
\hline$V=$ & 0.69 & \multicolumn{5}{|c|}{$=(D+)+(D-)$} \\
\hline$K=$ & 2.19 & \multicolumn{5}{|c|}{$=\left(n^{\wedge}(1 / 2)\right) * V$} \\
\hline
\end{tabular}

\section{Test V sur une direction attendue}

Comme la direction réelle ou attendue est connue (ici, $\left.\theta_{0}=150^{\circ}\right)$, il serait plus informatif de vérifier si les réponses des participants diffèrent ou non de celle-ci, plutôt que de constater seulement qu'elles ne sont pas uniformément distribuées. Un test $V$ peut être effectué en calculant le homeward component $(v)$ de l'échantillon, puis le paramètre $u$. Ici, $u$ excédant la valeur critique de 1.65 , on peut rejeter l'hypothèse d'uniformité et conclure que les réponses sont significativement agglomérées autour de la direction attendue de $150^{\circ}$.

\section{Test non paramétrique sur la déviation angulaire}

Des expériences de navigation et d'orientation (p. ex., celle de Montello et al., 1999) indiquant que les hommes réussissent mieux, en moyenne, que les femmes à ce type de tâches, l'expérimentatrice pourrait décider de comparer les participants des deux sexes. Ici, les participants 1 à 6 sont des hommes, 7 à 10 sont des femmes. Le test de Wallraff n'étant pas affecté par le fait que les échantillons sont de taille inégale, ceux-ci pourront être comparés avec ce test non paramétrique sur la dispersion. On calcule d'abord la déviation angulaire, arc $\cos \left(\phi_{\mathrm{i}}-\theta_{0}\right)$, de chaque réponse par rapport à $\theta_{0}\left(150^{\circ}\right)$, puis les participants sont placés en ordre croissant par rapport à celle-ci. Après avoir reçu un rang de 1 à 10, ils sont remis dans l'ordre initial afin de faciliter le calcul de $S_{1}$ et $S_{2}$. 
Figure 8 : Calcul des statistiques nécessaires à un test sur la déviation angulaire.

$\begin{array}{lcl}\mathrm{n} 1= & 6 & \\ \mathrm{n} 2= & 4 & \\ \text { Direction attendue }= & 150 & \\ \text { Direction attendue (rad) }= & 2.62 & =\text { RADIANS(Direction att } \\ \text { Déviation= } & - & =\text { ACOS(COS(Rép.(rad)- } \\ \text { Rang (méthode alternative) } & - & =\text { RANK(Dév. i; Dév. 1: [ } \\ \mathrm{S} 1= & 26 & =\text { SUM(RANG1: RANG6) } \\ \mathrm{S} 2= & 29 & =\text { SUM(RANG7:RANG10) } \\ \mathrm{U} 1= & 5 & =S 1-((\mathrm{n} 1 *(\mathrm{n}+1)) / 2) \\ \mathrm{U} 2= & 19 & =\mathrm{S} 2-((\mathrm{n} 2 *(\mathrm{n}+1)) / 2)\end{array}$

Le rang peut aussi être calculé sans trier les données: il s'agit de spécifier, pour la fonction RANK, la donnée dont on souhaite trouver le rang, la liste dont elle est issue et l'ordre de tri (une valeur supérieure à 0 pour un tri croissant). La valeur $U$ la plus faible est ici celle des hommes $\left(U_{1}\right)$. Comme elle excède la valeur critique de 3 (test unicaudal à $.05, ; n_{1}=6$ et $n_{2}=4$ ), on ne peut rejeter l'hypothèse nulle voulant qu'hommes et femmes ne diffèrent pas quant à leur déviation angulaire (ou performance d'orientation).

\section{Autres perspectives des statistiques circulaires}

L'étude des comportements d'orientation requiert, par la périodicité et l'arbitraire des mesures angulaires qu'elle utilise, l'emploi de méthodes statistiques adaptées aux données circulaires. Ce document fournit des instructions simples pour ce type de problèmes, du calcul de la moyenne jusqu'à l'inférence statistique. Cependant, les statistiques circulaires vont bien au-delà des mesures de direction: dans certaines situations, la distance parcourue est également importante. Il pourrait aussi s'agir de phénomènes cycliques pour lesquels la phase et l'amplitude sont mesurées. Les échantillons obtenus sont alors bivariés (une variable étant circulaire et l'autre, linéaire) et doivent être traités avec des techniques de corrélation linéaire-circulaire (abordées dans Batschelet, 1981). La corrélation entre deux variables circulaires, par exemple la direction du vent et la direction de vol d'un oiseau, peut également être étudiée. Pour des phénomènes aussi complexes ou pour produire des représentations graphiques circulaires, il devient indispensable de recourir à des logiciels statistiques spécialisés, par exemple Oriana pour Windows, CircStat pour S-Plus ou OAT-I et PhasePACK pour Matlab6.

\section{Références}

Batschelet, E. (1981). Circular statistics in biology. New York, NY: Academic Press.

Fisher, N. I. (1993). Statistical analysis of circular data. Cambridge, G.-B.: Cambridge University press. Jammalamadaka, S. R., SenGupta, A. (2001). Topics in circular

${ }^{6}$ OAT-I et PhasePACK sont disponibles gratuitement sur http://www.indiana.edu/ palab/links.htm statistics (Series on multivariate analysis, Vol. 5). Singapour: World Scientific.

Lindgren, B. W. (1968). Statistical Theory. Toronto, ON: Macmillan.

Montello, D. R., Lovelace, K. L., Golledge, R. G., \& Self, C. M., (1999). Sex-related differences and similarities in geographic and environmental spatial abilities. Annals of the Association of American Geographers, 89, 515-534.

Wallraff, H. G. (1979). Goal-oriented and compass-oriented movements of displaced homing pigeons after confinement in differentially shielded aviaries. Behavioral Ecology E Sociobiology, 5, 201-225.

Received August 14, 2005

Accepted September 26, 2005 